\title{
Boundedness of Area Functions Related to Schrödinger Operators and Their Commutators in Weighted Hardy Spaces
}

\author{
Lin Tang ${ }^{1, *}$, Jue Wang ${ }^{2}$ and Hua Zhu ${ }^{3}$ \\ ${ }^{1}$ LMAM, School of Mathematical Sciences, Peking University, Beijing 100871, China \\ 2 Department of Mathematics, Tufts University, Bromfield-Pearson Hall,503 Boston \\ Avenue Medford, MA 02155, USA \\ ${ }^{3}$ Beijing International Studies University, Beijing 100024, China
}

Received 14 July 2020; Accepted (in revised version) 21 July 2021

Dedicated to Prof. Shanzhen Lu with admiration on the occasion of his 80th birthday

\begin{abstract}
In this paper, we consider the area function $S_{Q}$ related to the Schrödinger operator $\mathcal{L}$ and its commutator $S_{Q, b}$, establish the boundedness of $S_{Q}$ from $H_{\rho}^{p}(w)$ to $L^{p}(w)$ or $W L^{p}(w)$, as well as the boundedness of $S_{Q, b}$ from $H_{\rho}^{1}(w)$ to $W L^{1}(w)$.
\end{abstract}

Key Words: Area functions, Schrödinger operator, weighted Hardy space.

AMS Subject Classifications: 42B25, 42B20

\section{Introduction}

Throughout this paper, $\mathcal{L}$ always denotes the following Schrödinger differential operator

$$
\mathcal{L}=-\Delta+V(x) \text { on } \mathbb{R}^{n}, \quad n \geq 3,
$$

where $V$ is a nonnegative potential belongs to reverse Hölder class $R H_{n / 2}$ (see Section 1.2).

The study of the Schrödinger operator $\mathcal{L}$ has recently attracted much attention, see [1, $2,4,5,12,19]$. In particular, Shen [12] proved the Schrödinger type operators, such as $\nabla(-\Delta+V)^{-1} \nabla, \nabla(-\Delta+V)^{-1 / 2},(-\Delta+V)^{-1 / 2} \nabla$ with $V \in R H_{n}$, and $(-\Delta+V)^{i \gamma}$ with $\gamma \in \mathbb{R}$ and $V \in R H_{n / 2}$, are standard Calderón-Zygmund operators.

In 2011, Bongioanni, etc. [1] introduced a new space of functions $B M O_{\theta}(\rho)$ as a generalization of the classical BMO space. They [2] also introduced a new weight class $A_{q}^{\rho, \theta}$ that

*Corresponding author. Email addresses: tanglin@math.pku.edu.cn (L. Tang), Jue.Wang@tufts.edu (J. Wang), zhuhua@pku.edu.cn (H. Zhu) 
locally behaves as Muckenhoupt's one and actually contains that. Both $B M O_{\theta}(\rho)$ and $A_{q}^{\rho, \theta}$ are associated with the potential $V$. The authors [1,2] also established $L^{p}\left(\mathbb{R}^{n}\right)(1<$ $p<\infty)$ boundedness for commutators of Riesz transforms associated with Schrödinger operators with $B M O_{\theta}(\rho)$ functions, and weighted boundedness for Riesz transforms, fractional integrals and Littlewood-Paley functions related to Schrödinger operator with $A_{q}^{\rho, \theta}$ weights. Recently, Tang, etc. [14-16] established weighted norm inequalities for some Schrödinger type operators, including commutators of Riesz transforms, fractional integrals, Littlewood-Paley functions and area functions related to Schrödinger operators, etc.

On the other hand, the function spaces related to $\mathcal{L}$ has attracted wide concern for years. In 1999, Dziubański and Zienkiewicz [4] defined the Hardy space related to $\mathcal{L}$, and gave some equivalent characterizations. In 2005, Dziubański, etc [5] defined the BMO space related to $\mathcal{L}$, and proved that it is the dual space of the above Hardy space. The weighted version of these theory have been also considered recently; see $[8,13]$. It should be pointed out that in $[8,13]$, the authors considered the weight functions belonging to Munckhoupt weight class. Very recently, Tang and Zhu [17] studied the properties of weighted Hardy spaces with $A_{q}^{\rho, \theta}$ weights.

In this paper, we continue to study weighted norm inequalities for area functions related to Schrödinger operators and their commutators. In fact, the weights we consider here are $A_{q}^{\rho, \theta}$ weights, and the weighted boundedness are of the type $H_{\rho}^{p}(\omega) \rightarrow L^{p}(\omega)$ or $W L^{p}(\omega)$, where $H_{\rho}^{p}(\omega)$ denotes weighted Hardy space related to $\rho$.

We first introduce some definitions. The area $S_{Q}$ function related to $\mathcal{L}$ is defined by

$$
S_{Q}(f)(x):=\left(\int_{0}^{\infty} \int_{|x-y|<t}\left|Q_{t}(f)(y)\right|^{2} \frac{d y d t}{t^{n+1}}\right)^{1 / 2},
$$

where

$$
\left(Q_{t} f\right)(x):=t^{2}\left(\left.\frac{d T_{s}}{d s}\right|_{s=t^{2}} f\right)(x), \quad T_{s}=e^{-s L}, \quad(x, t) \in \mathbb{R}_{+}^{n+1}=(0, \infty) \times \mathbb{R}^{n} .
$$

The commutator of $S_{Q}$ with $b \in B M O_{\theta}(\rho)$ is defined by

$$
S_{Q, b}(f)(x):=\left(\int_{0}^{\infty} \int_{|x-y|<t}\left|Q_{t}((b(x)-b(\cdot)) f)(y)\right|^{2} \frac{d y d t}{t^{n+1}}\right)^{1 / 2} .
$$

The main results of this paper are as follows.

Theorem 1.1. Let $q \geq 1$ and $w \in A_{q}^{\rho, \infty}$,

(i) if $\frac{n}{n+\delta_{0}}<p \leq 1$ and $1 \leq q \leq p\left(1+\frac{\delta_{0}}{n}\right)$, then for all $f \in H_{\rho}^{p}(\omega)$,

$$
\left\|S_{Q} f\right\|_{L^{p}(w)} \lesssim\|f\|_{H_{\rho}^{p}(\omega)}
$$


(ii) if $p=\frac{n}{n+\delta_{0}}$ and $w \in A_{1}^{\rho, \infty}$, then for all $f \in H_{\rho}^{p}(\omega)$,

$$
\left\|S_{Q} f\right\|_{W L^{p}(w)} \lesssim\|f\|_{H_{\rho}^{p}(\omega)},
$$

i.e., for all $\lambda>0$,

$$
\lambda \cdot w\left(\left\{x \in \mathbb{R}^{n}:\left|S_{Q}(f)(x)\right|>\lambda\right\}\right)^{1 / p} \lesssim\|f\|_{H_{\rho}^{p}(\omega)}
$$

where $\delta_{0}$ is defined in Lemma 2.4 and $H_{\rho}^{p}(\omega)$ is defined in Section 2.

For the commutator $S_{Q, b}$, we will prove the following result.

Theorem 1.2. If $w \in A_{1}^{\rho, \theta}, \theta \geq 0$, then for all $b \in B M O_{\theta}(\rho)$ and $f \in H_{\rho}^{1}(\omega)$,

$$
\left\|S_{Q, b} f\right\|_{W L^{1}(w)} \lesssim\|b\|_{B M O_{\theta}(\rho)}\|f\|_{H_{\rho}^{1}(\omega)} .
$$

The paper is organized as follows. In Section 2, we give some notations and basic results, most of which have been known before. In Section 3, we establish some useful lemmas for the main result. Finally, we prove Theorems 1.1 and 1.2 in Section 4.

\section{Preliminaries}

\subsection{Some basic notations}

Without opposite claim, $B$ always denotes a ball of $\mathbb{R}^{n}$ in this paper, i.e., $B=B(x, r):=$ $\left\{z \in \mathbb{R}^{n}:|z-x|<r\right\}$. The ball $\lambda B:=B(x, \lambda r)$ for $\lambda>0$ and $B=B(x, r)$.

Given a Lebesgue measurable set $E,|E|$ denotes the Lebesgue measure of $E$. If $|E|>0$, We write $f_{E}$ or $f_{E} f$ to denote $\frac{1}{|E|} \int_{E} f(x) d x$.

A weight $w(x)$ always means a nonnegative locally integrable function, which corresponds with a measure $d w:=w(x) d x$. Let $w(E):=\int_{E} d w$ denotes the weighted measure of E, and

$$
\|f\|_{L_{w}^{p}(X)}:=\left(\int_{\mathbb{R}^{n}}|f(y)|^{p} w(x) d x\right)^{1 / p} \quad \text { for } 0<p<\infty .
$$

The weighted $L^{p}(X)$ space $L_{w}^{p}(X)$ is defined as the set of all measurable functions $f$ satisfying $\|f\|_{L_{w}^{p}(X)<\infty}$. If $X$ can be omitted, we may write $L^{p}(w)$ or $L_{w}^{p}$ instead of $L_{w}^{p}(X)$ in the notations of both spaces and norms. The weighted weak $L^{p}$ norm $\|f\|_{W L_{\omega}^{p}(E)}$ is defined as

$$
\sup _{\lambda>0}\left\{\lambda \cdot w(\{x \in E:|f(x)|>\lambda\})^{1 / p}\right\},
$$

too. For $p \geq 1$, denote $p^{\prime}$ as the conjugate index of $p$, i.e., $1 / p+1 / p^{\prime}=1$.

Throughout this paper, it is usually supposed that $C$ denotes a positive constant independent of the main parameters involved, yet the value may differ from line to line. The notation $A \lesssim B$ means $A \leq C \cdot B$, and $A \gtrsim B$ the opposite. By $A \sim B$, we mean that $A \lesssim B$ and $A \gtrsim B$ simultaneously. If we want to emphasize that the the constant $C$ of a notation " $\lesssim$ " probably depends on a parameter, for example M, we may write " $\lesssim M$ ". So do the notations " $\gtrsim_{M}$ " and " $\sim_{M}$ ". 


\subsection{Reverse Hölder class}

We say a nonnegative locally $L^{q}$ integral function $V(x)$ on $\mathbb{R}^{n}$ belongs to the reverse Hölder class $R H_{q}(1<q<\infty)$, if and only if there exists $C>0$ such that the reverse Hölder inequality

$$
\left(\frac{1}{|B(x, r)|} \int_{B(x, r)} V^{q}(y) d y\right)^{1 / q} \leq C\left(\frac{1}{|B(x, r)|} \int_{B(x, r)} V(y) d y\right)
$$

holds for every $x \in \mathbb{R}^{n}$ and $0<r<\infty$, where $B(x, r)$ denotes the ball centered at $x$ with radius $r$. Apparently, provided $1<p<q<\infty, R H_{q}\left(\mathbb{R}^{n}\right) \subset R H_{p}\left(\mathbb{R}^{n}\right)$, so it is natural to define

$$
R H_{\infty}=\bigcap_{q>1} R H_{q} .
$$

For instance, if $V$ is a nonnegative polynomial, then $V \in B_{\infty}$. It is worth pointing out that if $V \in R H_{q}$ for some $q>1$, there exists $\epsilon>0$ depends on $n$ and the constant $C$ in (2.1) merely, such that $V \in R H_{q+\epsilon}$. Throughout this paper, we always assume that $0 \not \equiv V \in R H_{n / 2}$.

\subsection{Critical radius function $\rho(x)$ and the basic estimates of the kernel function $Q_{t}(\cdot, \cdot)$}

The critical radius function $\rho(x)$ is defined by

$$
\rho(x)=\sup _{r>0}\left\{r: \frac{1}{r^{n-2}} \int_{B(x, r)} V(y) d y \leq 1\right\} .
$$

Obviously, $0<\rho(x)<\infty$ if $V \neq 0$. In particular, $\rho(x) \sim 1$ with $V=1$ and $\rho(x) \sim$ $(1+|x|)^{-1}$ with $V=|x|^{2}$.

The next lemma has basic importance regarding $\rho(x)$.

Lemma 2.1 ([12]). There exist constants $l_{0}>0$ and $C_{0}>1$ such that

$$
\frac{1}{C_{0}}\left(1+\frac{|x-y|}{\rho(x)}\right)^{-l_{0}} \leq \frac{\rho(y)}{\rho(x)} \leq C_{0}\left(1+\frac{|x-y|}{\rho(x)}\right)^{l_{0} /\left(l_{0}+1\right)} .
$$

From this Lemma, it is easy to prove the next corollary.

Corollary 2.1. If $\rho(z) \gtrsim|x-z|$,

$$
\rho(x) \sim \rho(z) .
$$

If $\rho(z) \lesssim|x-z|$

$$
\left(\frac{\rho(z)}{|x-z|}\right)^{l_{0}+1} \lesssim \frac{\rho(x)}{|x-z|} \lesssim\left(\frac{\rho(z)}{|x-z|}\right)^{1 /\left(l_{0}+1\right)}
$$

The constant $l_{0}$ refers to Lemma 2.1 . 
Remark 2.1. For $B=B\left(x_{0}, r\right)$, we always denote $\Psi(B)=1+r / \rho\left(x_{0}\right)$ in this paper.

We write $Q_{t}(\cdot, \cdot)$ as the integral kernel of the operator $Q_{t}$ defined at (1.1). The following estimates of $Q_{t}(\cdot, \cdot)$ is basic and crucial in this paper.

Lemma 2.2 ([5]). There exist constants $c>0,0<\delta_{0} \leq 1$ such that for each $N>0$, both of the estimates

(i) $\left|Q_{t}(y, z)\right| \lesssim_{N} t^{-n}\left(1+\frac{t}{\rho(y)}+\frac{t}{\rho(z)}\right)^{-N} \exp \left(-\frac{c|y-z|^{2}}{t^{2}}\right)$;

(ii) $\left|Q_{t}(y, z)-Q_{t}(y, x)\right| \lesssim_{N}\left(\frac{|x-z|}{t}\right)^{\delta_{0}} t^{-n}\left(1+\frac{t}{\rho(y)}+\frac{t}{\rho(z)}\right)^{-N} \exp \left(-\frac{c|y-z|^{2}}{t^{2}}\right)$;

hold for all $x, y, z \in \mathbb{R}^{n}$ with $|x-z| \leq t$.

\section{$2.4 A_{q}^{\rho, \theta}$ weights}

Bongioanni, etc, in [1] introduced a new kind of weight class $A_{q}^{\rho, \theta}$, which associates with the potential $V$ by the critical radius function $\rho(x)$.

Definition 2.1 ([2]). For $0 \leq \theta<\infty, 1<q<\infty$, we say a weight $w(x)$ belongs to the $A_{q}^{\rho, \theta}$ class, if it satisfies the so-called $A_{q}^{\rho, \theta}$ condition

$$
\left(f_{B} w\right)^{1 / q}\left(f_{B} w^{-\frac{q^{\prime}}{q}}\right)^{1 / q^{\prime}} \lesssim \Psi(B)^{\theta}
$$

for all balls $B=B\left(x_{0}, r\right)$. As for $q=1$, the $A_{1}^{\rho, \theta}$ condition is defined as

$$
M_{V}^{\theta}(w)(x) \lesssim w(x), \quad \text { a.e. } x \in \mathbb{R}^{n},
$$

for all balls $B=B\left(x_{0}, r\right)$, where

$$
M_{V}^{\theta} f(x):=\sup _{x \in B} \frac{1}{\Psi(B)^{\theta}} f_{B}|f| .
$$

In this paper, we let

$$
A_{q}^{\rho, \infty}=\bigcup_{\theta>0} A_{q}^{\rho, \theta} \quad \text { for } \quad q \geq 1 .
$$

Remark 2.2. (i) In previous definitions, balls $B$ can be replaced by cubes $Q$, since $\Psi(B)^{\theta} \leq$ $\Psi(\lambda B)^{\theta} \leq \lambda^{\theta} \Psi(B)^{\theta}$ for all balls $B$ and all $\lambda>1$.

(ii) The infimum $C$ of all feasible constants related to " $\lesssim$ " in formula (2.3) or (2.4) is called the $A_{q}^{\rho, \theta}$ weight constant or $A_{1}^{\rho, \theta}$ weight constant of $w$, where $C$ is independent of the selection of $B$. 
Since $\Psi(B) \geq 1$, the class $A_{q}^{\rho, \theta}$ increases when $\theta$ increases, so we define

$$
A_{q}^{\rho, \infty}:=\bigcup_{\theta \geq 0} A_{q}^{\rho, \theta}
$$

naturally. It is also obvious that $A_{p} \subset A_{q}^{\rho, \theta}$ for $1 \leq p<\infty$, where $A_{q}$ denote the classical Muckenhoupt weights; see $[6,10]$. We will see that $A_{q} \varsubsetneqq A_{q}^{\rho, \theta}$ for $1 \leq p<\infty$ in some cases. In fact, let $\theta>0$ and $0 \leq \gamma \leq \theta$, it is easy to check that

$$
w(x)=(1+|x|)^{-(n+\gamma)} \notin A_{\infty}=\bigcup_{p \geq 1} A_{q}
$$

and $d w=w(x) d x$ does not satisfy the doubling condition, but

$$
w(x)=(1+|x|)^{-(n+\gamma)} \in A_{1}^{\rho, \theta}
$$

provided $V=1$ and thus

$$
\Psi_{\theta}\left(B\left(x_{0}, r\right)\right) \sim(1+r)^{\theta} .
$$

According to the definition, the difference between $A_{q}$ weight and $A_{q}^{\rho, \theta}$ weight is just on $\rho(x)$, so for such balls $B=B\left(x_{0}, r\right)$ with $r \lesssim \rho\left(x_{0}\right), A_{q}^{\rho, \theta}$ weights behave as same as $A_{q}$ weights.

When $V=0$ and $\theta=0, M_{0,0} f(x)$ is actually the standard Hardy-Littlewood maximal function $M f(x)$. Obviously,

$$
|f(x)| \leq M_{V}^{\theta} f(x) \leq M f(x) \quad \text { for a.e. } x \in \mathbb{R}^{n} \text { and } \theta \geq 0 .
$$

Similarly as the condition of $A_{p}$ weight, the following basic properties of $A_{q}^{\rho, \theta}$ weights hold, whose proofs are really elementary by the definition and so that to be omitted.

Lemma 2.3 ([14]). Suppose $1 \leq q<p \leq \infty$ and $\theta \geq 0$, we have

(i) $A_{q}^{\rho, \theta} \subset A_{p}^{\rho, \theta}$;

(ii) $w \in A_{p}^{\rho, \theta}$ if and only if $w^{-\frac{1}{p-1}} \in A_{p^{\prime}}^{\rho, \theta}$;

(iii) If $w \in A_{q}^{\rho, \theta}$, then for all balls $B$ and measurable set $E \subset B$,

$$
\frac{w(B)}{w(E)} \lesssim\left(\frac{|B|}{|E|}\right)^{q} \cdot \Psi(B)^{\theta q}
$$

As a deep result, reverse Hölder inequality is of remarkable importance in the theory of $A_{q}$ weight, from which we can acquire the property $A_{\infty}$ weight condition for $A_{q}$ weights. Similarly, such properties can be paralleled to $A_{q}^{\rho, \theta}$ weights with minor difference. 
Proposition 2.1 ([2]). Let $1 \leq q<\infty, \theta \geq 0, w \in A_{q}^{\rho, \theta}$.

(i) (Reverse Hölder property). There exist constants $\epsilon, \eta_{0}>0$, such that

$$
\left(f_{B} w^{1+\epsilon}\right)^{\frac{1}{1+\epsilon}} \lesssim\left(f_{B} w\right) \Psi(B)^{\eta_{0}}
$$

for all balls $B$. It is worth emphasizing that the constant $\epsilon$ depends on $w(x)$ only concerning the $A_{q}^{\rho, \theta}$ constant of $w(x)$, while $\eta_{0}$ can depend on $n, q, \theta$ and $l_{0}$ (as in Lemma 2.1) only.

(ii) If $q>1$, there exists $p \in(1, q)$ and $\theta^{\prime} \geq 0$ satisfying $w \in A_{p}^{\rho, \theta^{\prime}}$. As in (i), p depends on $w(x)$ only concerning the $A_{q}^{\rho, \theta}$ constant of $w(x)$.

(iii) There exists a constant $\delta=\frac{\epsilon}{1+\epsilon} \in(0,1)$ such that

$$
\frac{w(E)}{w(B)} \lesssim\left(\frac{|E|}{|B|}\right)^{\delta} \Psi(B)^{\eta_{0}}
$$

holds for all balls $B$ and measurable set $E \subset B$, where the constants $\epsilon, \eta_{0}$ have appeared in (i).

Proof. For (i) and (ii), we refer to the proof of Lemma 5 And Proposition 5 in [2], so we will just prove (iii).

By Hölder inequality and (i), for all balls B and measurable set $E \subset B$,

$$
\begin{aligned}
w(E) & =\int_{B} w 1_{E} \leq\left(\int_{B} w^{1+\epsilon}\right)^{\frac{1}{1+\epsilon}}|E|^{\frac{\epsilon}{1+\epsilon}} \\
& \lesssim|B|^{-\frac{\epsilon}{1+\epsilon}} w(B) \Psi(B)^{\eta_{0}}|E|^{\frac{\epsilon}{1+\epsilon}} .
\end{aligned}
$$

Thus, (iii) holds.

\section{$2.5 B M O_{\theta}(\rho)$ space}

Bongioanni, etc. [1] introduced the following new space $B M O_{\theta}(\rho)$ as the substitution of classical $B M O$ space.

Definition 2.2 ([1]). Let $\theta \geq 0, \rho$ is defined in (2.2). For a locally integrable function $f(x)$, the $B M O_{\theta}(\rho)$ norm is defined by

$$
\|f\|_{B M O_{\theta}(\rho)}=\sup _{B \subset \mathbb{R}^{n}} \frac{1}{\Psi(B)^{\theta}} f_{B}\left|f(x)-f_{B}\right| d x<\infty,
$$

where $B$ is a ball. The following spaces are defined

$$
\begin{aligned}
& B M O_{\theta}(\rho):=\left\{f \in L_{l o c}\left(\mathbb{R}^{n}\right):\|f\|_{B M O_{\theta}(\rho)}<\infty\right\}, \\
& B M O_{\infty}(\rho):=\bigcup_{\theta>0} B M O_{\theta}(\rho) .
\end{aligned}
$$


Clearly from the definition, if $\theta=0, B M O_{0}(\rho)$ is the classical $B M O$ in actual. And the space $B M O_{\theta}(\rho)$ enlarges while $\theta$ increases. Bongioanni, etc. [1] also proved the following result.

Lemma 2.4 ([1]). Let $s \geq 1$ and $b \in B M O_{\theta}(\rho)$, then

$$
\left(f_{B}\left|b-b_{B}\right|^{s}\right)^{1 / s} \lesssim\|b\|_{B M O_{\theta}(\rho)} \Psi(B)^{\theta^{\prime}}
$$

for all balls $B$, where $\theta^{\prime}=\left(l_{0}+1\right) \theta$ and $l_{0}$ correspond with that in Lemma 2.1.

Corollary 2.2 ([1]). Let $s \geq 1$ and $b \in B M O_{\theta}(\rho)$, then

$$
\left(f_{2^{k} B}\left|b-b_{B}\right|^{s}\right)^{1 / s} \lesssim k\|b\|_{B M O_{\theta}(\rho)} \Psi\left(2^{k} B\right)^{\theta^{\prime}}
$$

for all balls $B$ and all $k \in \mathbb{N}^{*}$, with $\theta^{\prime}$ as in Lemma 2.4.

By Lemma 2.4, Tang [14] established the John-Nirenberg type inequality for $B M O_{\theta}(\rho)$ functions as well.

Proposition 2.2 ([14]). If $b \in B M O_{\theta}(\rho)$, then there exists a constant $c>0$ such that both of the inequalities

(i)

$$
\frac{1}{|B|}\left|\left\{x \in B:\left|b(x)-b_{B}\right|>\lambda\right\}\right| \lesssim \exp \left(\frac{-c \lambda}{\|b\|_{B M O_{\theta}(\rho)} \Psi(B)^{\theta^{\prime}}}\right)
$$

(ii)

$$
\sup _{B} f_{B} \exp \left(\frac{c\left|f(x)-b_{B}\right|}{\|b\|_{B M O_{\theta}(\rho)} \Psi(B)^{\theta^{\prime}}}\right) d x \lesssim 1
$$

hold for all balls $B$ and all $\lambda>0$, where $\theta^{\prime}$ corresponds with that in Lemma 2.4.

\subsection{Weighted Hardy spaces $H_{\mathcal{L}}^{p}(w)$ and $H_{\rho}^{p}(\omega)$}

The two weighted Hardy spaces $H_{\mathcal{L}}^{p}(w)$ and $H_{\rho}^{p}(\omega)$ are defined and studied by Tang and Zhu in [17]. More precisely, let

$$
\begin{aligned}
& H_{\mathcal{L}}^{p}(w):=\left\{f \in \mathcal{S}^{\prime}:\left\|T^{*} f\right\|_{\left.L_{w}^{p}<\infty\right\},} \quad \text { where } T^{*} f(x)=\sup _{t>0}\left|e^{-t \mathcal{L}} f(x)\right|,\right. \\
& H_{\rho}^{p}(\omega):=\left\{f \in \mathcal{S}^{\prime}:\left\|\widetilde{T}^{*} f\right\|_{L_{w}^{p}}<\infty\right\}, \quad \text { where } \widetilde{T}^{*} f(x)=\sup _{0<t<\rho(x)}\left|e^{-t \triangle} f(x)\right|,
\end{aligned}
$$

where the symbols $\mathcal{S}^{\prime}$ is the dual space of Schwartz functions. 
Moreover, the norm of the spaces $H_{\mathcal{L}}^{p}(w)$ and $H_{\rho}^{p}(\omega)$ is defined respectively

$$
\|f\|_{H_{\mathcal{L}}^{p}(w)}:=\left\|T^{*} f\right\|_{L_{w}^{p},} \quad\|f\|_{H_{\rho}^{p}(\omega)}:=\left\|\widetilde{T}^{*} f\right\|_{L_{w}^{p}} .
$$

We remark that when $\omega=1$ and $p=1$, the $H_{\rho}^{p}(\omega)$ space has been studied by Yang and Zou in [18], and the $H_{\mathcal{L}}^{p}(\omega)$ space with $\omega=1$ has been studied by Dziubański, etc. [4,5].

As in the classical condition, we can also give the theory of atomic decomposition for $H_{\mathcal{L}}^{p}(w)$ and $H_{\rho}^{p}(\omega)$. We first introduce some definitions.

From Lemma 2.1, for any given $w \in A_{q}^{\rho, \infty}$, define the critical index of $w$ by

$$
q_{w}:=\inf \left\{p \in[1, \infty): w \in A_{p}^{\rho, \infty}\right\} .
$$

Let $\omega \in A_{\infty}^{\rho, \infty}=\bigcup_{q>1} A_{q}^{\rho, \infty}$ and $q_{\omega}$ be as in (2.5). A triplet $(p, q, s)_{\omega}$ is called to be admissible, if $p \in(0,1], q \in\left(q_{\omega}, \infty\right]$ and $s \in \mathbb{N}$ with $s \geq\left[n\left(\frac{q_{\omega}}{p}-1\right)\right]$. A function $a$ on $\rho$ is said to be a $(p, q, s)_{\omega}$-atom associated with a ball $B=B\left(x_{0}, r\right)$, if

(i) supp $a \subset B$ with $r \leq 2 \rho\left(x_{0}\right)$;

(ii) $\|a\|_{L_{w}^{q}} \leq w(B)^{1 / q-1 / p}$;

(iii) If $\gamma_{0} r<\rho\left(x_{0}\right)$, then

$$
\int_{\mathbb{R}^{n}} a(x) x^{\alpha} d x=0 \quad \text { for } \quad \alpha \in(\{0\} \bigcup \mathbb{N})^{n} \quad \text { with }|\alpha| \leq s .
$$

The constant $\gamma_{0}=4 n C_{0}^{2}$, where $C_{0}$ appears in Lemma 2.1.

Moreover, we call $a$ is a $(p, q)_{\omega}$ single atom if

$$
\|a\|_{L_{w}^{q}\left(\mathbb{R}^{n}\right)} \leq\left[w\left(\mathbb{R}^{n}\right)\right]^{1 / q-1 / p}
$$

provided that $w\left(\mathbb{R}^{n}\right)<\infty$.

Let $\omega \in A_{\infty}^{\rho, \infty}$ and $(p, q, s)_{\omega}$ be an admissible triplet. The weighted atomic local Hardy space $h_{\rho}^{p, q, s}(\omega)$ is defined to be the set of all $f \in \mathcal{S}^{\prime}$ satisfying that $f=\sum_{i=0}^{\infty} \lambda_{i} a_{i}$ in $\mathcal{S}^{\prime}$, where $\left\{\lambda_{i}\right\}_{i \in \mathbb{N}_{0}} \subset \mathbb{C}, \sum_{i=0}^{\infty}\left|\lambda_{i}\right|^{p}<\infty$ and $\left\{a_{i}\right\}_{i \in \mathbb{N}}$ are $(p, q, s)_{\omega}$-atom and $a_{0}$ is a $(p, q)_{\omega}$ single atom. Moreover, the quasi-norm of $f \in H_{\rho}^{p, q, s}(\omega)$ is defined by

$$
\|f\|_{H_{\rho}^{p, q, s}(\omega)} \equiv \inf \left\{\left[\sum_{i=0}^{\infty}\left|\lambda_{i}\right|^{p}\right]^{1 / p}\right\}
$$

where the infimum is taken over all the decompositions of $f$ as above.

It is easy to see that if the triplets $(p, q, s)_{\omega}$ and $(p, \bar{q}, \bar{s})_{\omega}$ are admissible and satisfy $\bar{q} \leq q$ and $\bar{s} \leq s$, then $(p, q, s)_{\omega}$-atoms are $(p, \bar{q}, \bar{s})_{\omega}$-atoms, which further implies that

$$
H_{\rho}^{p, q, s}(\omega) \subset H_{\rho}^{p, \bar{q}, \bar{s}}(\omega)
$$

and the inclusion is continuous.

Recently, Tang and Zhu [17] proved the following results. 
Theorem 2.1. Let $0 \not \equiv V \in R H_{n / 2}, w \in A_{\infty}^{\rho, \infty}, q_{w}$ be as in (2.5) and $(p, q, s)_{\omega}$ be an admissible triplet.

(i) If $0<p \leq 1$ and $q \in\left(q_{w}, \infty\right)$, then

$$
\|f\|_{H_{\rho}^{p}(\omega)} \sim\|f\|_{H_{\rho}^{p, q, s}(\omega)} .
$$

(ii) If $\frac{n}{n+\delta_{0}}<p \leq 1$ and $q \in\left(q_{w}, p\left(1+\frac{\delta_{0}}{n}\right)\right)$, then

$$
\|f\|_{H_{\rho}^{p}(\omega)} \sim\|f\|_{H_{\mathcal{L}}^{p}(w)} \sim\|f\|_{H_{\rho}^{p, q, s}(\omega)} .
$$

Let $w \in A_{\infty}^{\rho, \infty}$ and $(p, q, s)_{w}$ be an admissible triplet. Denote by $H_{\rho, f i n}^{p, q, s}(\omega)$ the vector space of all finite linear combination of $(p, q, s)_{\omega}$ atoms and single atom, and the norm of $f$ in $H_{\rho, f i n}^{p, q, s}(\omega)$ is defined by

$$
\|f\|_{H_{\rho, f i n}^{p, q, s}(\omega)}:=\inf \left\{\left(\sum_{j=0}^{k}\left|\lambda_{j}\right|^{p}\right)^{1 / p}: f=\sum_{j=0}^{k} \lambda_{j} a_{j}, k \in \mathbb{N}\right\},
$$

where $a_{j}$ are all $(p, q, s)_{\omega}$ atoms and $a_{0}$ is a $(p, q)_{\omega}$ single atom.

Obviously, for any admissible triplet $(p, q, s)_{\omega}$ atom and $(p, q)_{\omega}$ single atom, the set $H_{\rho, f i n}^{p, q, s}(\omega)$ is dense in $H_{\rho}^{p, q, s}(\omega)$ with respect to the quasi-norm $\|\cdot\|_{H_{\rho, f i n}^{p, q, s}(\omega)}$.

Theorem 2.2. Let $w \in A_{\infty}^{\rho, \infty}, q_{w}$ be as in (2.5) and $(p, q, s)_{\omega}$ be an admissible triplet. If $q \in$ $\left(q_{w}, \infty\right)$, then $\|\cdot\|_{H_{\rho, f i n}^{p, q, s}(\omega)}$ and $\|\cdot\|_{H_{\rho}^{p}(\omega)}$ are equivalent quasi-norms on $H_{\rho, f i n}^{p, q, s}(\omega)$.

\section{Basic estimates for $S_{Q}$ and some lemmas}

Applying some results in Section 2.2, we establish an estimate of $S_{Q} a(x)$ for $(p, q)_{\omega}:=$ $(p, q, 0)_{\omega}$ atoms $a(x)$ as a basis of the proof of our main results.

Proposition 3.1. Let $1 \leq q<\infty, w \in A_{\infty}^{\rho, \infty}$. For any $(p, q)_{\omega}$ atoms a associated with $B=$ $B\left(x_{0}, r\right)$ and all $x \in(4 B)^{c}$,

Case 1. if $\gamma_{0} r<\rho\left(x_{0}\right)$ and $\left|x-x_{0}\right| \leq 2 \rho\left(x_{0}\right)$,

$$
S_{Q} a(x) \lesssim\|a\|_{L^{1}} \frac{r^{\delta_{0}}}{\left|x-x_{0}\right|^{n+\delta_{0}}}
$$

Case 2. if $\gamma_{0} r<\rho\left(x_{0}\right)$ and $\left|x-x_{0}\right| \geq \rho\left(x_{0}\right)$, for each $M>0$,

$$
S_{Q} a(x) \lesssim_{M}\|a\|_{L^{1}} \frac{r^{\delta_{0}}}{\left|x-x_{0}\right|^{n+\delta_{0}}}\left(\frac{\rho\left(x_{0}\right)}{\left|x-x_{0}\right|}\right)^{M},
$$


Case 3. if $\gamma_{0} r \geq \rho\left(x_{0}\right)$, for each $M>0$,

$$
S_{Q} a(x) \lesssim_{M}\|a\|_{L^{1}} \frac{r^{M}}{\left|x-x_{0}\right|^{n+M}}
$$

The constant $\delta_{0}$ corresponds as in Lemma 2.2(ii).

Proof. In the proof, we will use the elementary inequality $e^{-c t^{2}} \lesssim_{c, M}(1+|t|)^{-M}$ (for all $t \in \mathbb{R}$ where $c>0, M \geq 0$ are both constants) and Lemma 2.2 repeatedly. It is obvious from Theorem 2.1 that $a(x)$ has vanishing property in cases 1 and 2 .

For case 1 , we have

$$
\begin{aligned}
S_{Q} a(x)^{2} & =\int_{0}^{\infty} \int_{|x-y|<t}\left|\int_{B}\left(Q_{t}(y, z)-Q_{t}\left(y, x_{0}\right)\right) a(z) d z\right|^{2} \frac{d y d t}{t^{n+1}} \\
& =\left(\int_{0}^{r} \cdots\right)+\left(\int_{r}^{\frac{\left|x-x_{0}\right|}{2}} \cdots\right)+\left(\int_{\frac{\left|x-x_{0}\right|}{2}}^{\infty} \cdots\right) \\
& =:\left(I_{1}\right)^{2}+\left(I_{2}\right)^{2}+\left(I_{3}\right)^{2} .
\end{aligned}
$$

For $I_{1}, y \in B(x, t) \subset B(x, r)$ and $z \subset B\left(x_{0}, r\right)$, so $|y-z| \sim\left|x-x_{0}\right|$. Then we have

$$
\begin{aligned}
I_{1} & =\left(\int_{0}^{r} \int_{B(x, t)}\left|\int_{B} Q_{t}(y, z) a(z) d z\right|^{2} \frac{d y d t}{t^{n+1}}\right)^{\frac{1}{2}} \\
& \lesssim\left(\int_{0}^{r} \int_{B(x, t)}\left(\int_{B} t^{-n}\left(\frac{\left|y-x_{0}\right|}{t}\right)^{-n-\delta_{0}}|a(z)| d z\right)^{2} \frac{d y d t}{t^{n+1}}\right)^{\frac{1}{2}} \\
& \lesssim\left|x-x_{0}\right|^{-n-\delta_{0}}\|a\|_{L^{1}}\left(\int_{0}^{r} t^{2 \delta_{0}-1} d t\right)^{\frac{1}{2}} \sim\|a\|_{L^{1}} \frac{r^{\delta_{0}}}{\left|x-x_{0}\right|^{n+\delta_{0}}} .
\end{aligned}
$$

For $I_{2}$, since $\left|z-x_{0}\right|<r<t$, by Lemma 2.2(ii), we have

$$
\begin{aligned}
I_{2} & =\left(\int_{r}^{\frac{\left|x-x_{0}\right|}{2}} \int_{B(x, t)}\left|\int_{B}\left(Q_{t}(y, z)-Q_{t}\left(y, x_{0}\right)\right) a(z) d z\right|^{2} \frac{d y d t}{t^{n+1}}\right)^{\frac{1}{2}} \\
& \lesssim\left(\int_{r}^{\frac{\left|x-x_{0}\right|}{2}} \int_{B(x, t)}\left(\int_{B} t^{-n}\left(\frac{\left|z-x_{0}\right|}{t}\right)^{\delta_{0}}\left(\frac{\left|y-x_{0}\right|}{t}\right)^{-n-\delta_{0}-1}|a(z)| d z\right)^{2} \frac{d y d t}{t^{n+1}}\right)^{\frac{1}{2}} \\
& \lesssim \frac{r^{\delta_{0}}}{\left|x-x_{0}\right|^{n+\delta_{0}+1}}\|a\|_{L^{1}}\left(\int_{0}^{\frac{\left|x-x_{0}\right|}{2}} t d t\right)^{\frac{1}{2}} \sim\|a\|_{L^{1}} \frac{r^{\delta_{0}}}{\left|x-x_{0}\right|^{n+\delta_{0}}} .
\end{aligned}
$$


For $I_{3}$, we get

$$
\begin{aligned}
I_{3} & =\left(\int_{\frac{\left|x-x_{0}\right|}{2}}^{\infty} \int_{B(x, t)}\left|\int_{B}\left(Q_{t}(y, z)-Q_{t}\left(y, x_{0}\right)\right) a(z) d z\right|^{2} \frac{d y d t}{t^{n+1}}\right)^{\frac{1}{2}} \\
& \lesssim\left(\int_{\frac{\left|x-x_{0}\right|}{2}}^{\infty} \int_{B(x, t)}\left(\int_{B} t^{-n}\left(\frac{\left|z-x_{0}\right|}{t}\right)^{\delta_{0}}|a(z)| d z\right)^{2} \frac{d y d t}{t^{n+1}}\right)^{\frac{1}{2}} \\
& \lesssim r^{\delta_{0}}\|a\|_{L^{1}}\left(\int_{\frac{\left|x-x_{0}\right|}{2}}^{\infty} \frac{d t}{t^{2 n+2 \delta_{0}+1}}\right)^{\frac{1}{2}} \sim\|a\|_{L^{1}} \frac{r^{\delta_{0}}}{\left|x-x_{0}\right|^{n+\delta_{0}}} .
\end{aligned}
$$

Thus, Case 1 has been solved.

For Case 2, we have

$$
\begin{aligned}
S_{Q} a(x)^{2} & =\int_{0}^{\infty} \int_{|x-y|<t}\left|\int_{B}\left(Q_{t}(y, z)-Q_{t}\left(y, x_{0}\right)\right) a(z) d z\right|^{2} \frac{d y d t}{t^{n+1}} \\
& =\left(\int_{0}^{r} \cdots\right)+\left(\int_{r}^{\frac{\left|x-x_{0}\right|}{2}} \cdots\right)+\left(\int_{\frac{\left|x-x_{0}\right|}{2}}^{\infty} \cdots\right) \\
& =:\left(I I_{1}\right)^{2}+\left(I I_{2}\right)^{2}+\left(I I_{3}\right)^{2},
\end{aligned}
$$

and $M$ is an arbitrary positive constant.

For $I I_{1},|y-z| \sim\left|x-x_{0}\right|$ as well, then

$$
\begin{aligned}
& I I_{1}=\left(\int_{0}^{r} \int_{B(x, t)}\left|\int_{B} Q_{t}(y, z) a(z) d z\right|^{2} \frac{d y d t}{t^{n+1}}\right)^{\frac{1}{2}} \\
& \lesssim_{M}\left(\int_{0}^{r} \int_{B(x, t)}\left(\int_{B} t^{-n}\left(\frac{|y-z|}{t}\right)^{-M-n}|a(z)| d z\right)^{2} \frac{d y d t}{t^{n+1}}\right)^{\frac{1}{2}} \\
& \lesssim_{M}\left|x-x_{0}\right|^{-M-n}\|a\|_{L^{1}}\left(\int_{0}^{r} t^{2 M-1} d t\right)^{\frac{1}{2}} \sim\|a\|_{L^{1}} \frac{r^{M}}{\left|x-x_{0}\right|^{M+n}} .
\end{aligned}
$$

Given that $r \lesssim \rho\left(x_{0}\right)$, we have already obtained the required estimate for $I I_{1}$. For $I I_{2},\left|y-x_{0}\right| \sim\left|x-x_{0}\right|$ as well, then

$$
\begin{aligned}
I I_{2} & =\left(\int_{r}^{\frac{\left|x-x_{0}\right|}{2}} \int_{B(x, t)}\left|\int_{B}\left(Q_{t}(y, z)-Q_{t}\left(y, x_{0}\right)\right) a(z) d z\right|^{2} \frac{d y d t}{t^{n+1}}\right)^{\frac{1}{2}} \\
& \lesssim M\left(\int_{r}^{\frac{\left|x-x_{0}\right|}{2}} \int_{B(x, t)}\right.
\end{aligned}
$$




$$
\begin{aligned}
& \left.\times\left(\int_{B} t^{-n}\left(\frac{\left|z-x_{0}\right|}{t}\right)^{\delta_{0}}\left(\frac{\rho\left(x_{0}\right)}{t}\right)^{M}\left(\frac{\left|y-x_{0}\right|}{t}\right)^{-M-n-\delta_{0}-1}|a(z)| d z\right)^{2} \frac{d y d t}{t^{n+1}}\right)^{\frac{1}{2}} \\
\lesssim & \frac{r^{\delta_{0}} \rho\left(x_{0}\right)^{M}}{\left|x-x_{0}\right|^{M+n+\delta_{0}+1}}\|a\|_{L^{1}}\left(\int_{0}^{\frac{\left|x-x_{0}\right|}{2}} t d t\right)^{\frac{1}{2}} \sim\|a\|_{L^{1}} \frac{r^{\delta_{0}}}{\left|x-x_{0}\right|^{n+\delta_{0}}}\left(\frac{\rho\left(x_{0}\right)}{\left|x-x_{0}\right|}\right)^{M} .
\end{aligned}
$$

For $I_{3}$, we have

$$
\begin{aligned}
I I_{3} & =\left(\int_{\frac{\left|x-x_{0}\right|}{2}}^{\infty} \int_{B(x, t)}\left|\int_{B}\left(Q_{t}(y, z)-Q_{t}\left(y, x_{0}\right)\right) a(z) d z\right|^{2} \frac{d y d t}{t^{n+1}}\right)^{\frac{1}{2}} \\
& \lesssim_{M}\left(\int_{\frac{\left|x-x_{0}\right|}{2}}^{\infty} \int_{B(x, t)}\left(\int_{B} t^{-n}\left(\frac{\left|z-x_{0}\right|}{t}\right)^{\delta_{0}}\left(\frac{\rho\left(x_{0}\right)}{t}\right)^{M}|a(z)| d z\right)^{2} \frac{d y d t}{t^{n+1}}\right)^{\frac{1}{2}} \\
& \lesssim_{M} r^{\delta_{0}} \rho\left(x_{0}\right)^{M}\|a\|_{L^{1}}\left(\int_{\frac{\left|x-x_{0}\right|}{2}}^{\infty} \frac{d t}{t^{2 M+2 \delta_{0}+2 n+1}}\right)^{\frac{1}{2}} \sim\|a\|_{L^{1}} \frac{r^{\delta_{0}}}{\left|x-x_{0}\right|^{n+\delta_{0}}}\left(\frac{\rho\left(x_{0}\right)}{\left|x-x_{0}\right|}\right)^{M} .
\end{aligned}
$$

Thus, Case 2 has already been solved. Finally we turn to consider Case 3 in which $r \sim$ $\rho\left(x_{0}\right)$. As in previous cases,

$$
\begin{aligned}
S_{Q} a(x)^{2} & =\int_{0}^{\infty} \int_{|x-y|<t} \mid \int_{B}\left(\left.Q_{t}(y, z) a(z) d z\right|^{2} \frac{d y d t}{t^{n+1}}\right. \\
& =\left(\int_{0}^{r} \cdots\right)+\left(\int_{r}^{\frac{\left|x-x_{0}\right|}{2}} \cdots\right)+\left(\int_{\frac{\left|x-x_{0}\right|}{2}}^{\infty} \cdots\right) \\
& :=\left(I I I_{1}\right)^{2}+\left(I I I_{2}\right)^{2}+\left(I I I_{3}\right)^{2},
\end{aligned}
$$

where $M$ is an arbitrary positive constant.

For $I I I_{1}$, the estimation is the same as that in $I I_{1}$ exactly, namely

$$
I I I_{1} \lesssim M\|a\|_{L^{1}} \frac{r^{M}}{\left|x-x_{0}\right|^{n+M}} .
$$

For $I I I_{2},|y-z| \sim\left|x-x_{0}\right|$ as well. So we have

$$
\begin{aligned}
I I I_{2} & =\left(\int_{r}^{\frac{\left|x-x_{0}\right|}{2}} \int_{B(x, t)}\left|\int_{B} Q_{t}(y, z) a(z) d z\right|^{2} \frac{d y d t}{t^{n+1}}\right)^{\frac{1}{2}} \\
& \lesssim M\left(\int_{r}^{\frac{\left|x-x_{0}\right|}{2}} \int_{B(x, t)}\left(\int_{B} t^{-n}\left(\frac{\rho\left(x_{0}\right)}{t}\right)^{M}\left(\frac{|y-z|}{t}\right)^{-M-n-1}|a(z)| d z\right)^{2} \frac{d y d t}{t^{n+1}}\right)^{\frac{1}{2}} \\
& \lesssim M \frac{\rho\left(x_{0}\right)^{M}}{\left|x-x_{0}\right|^{M+n+1}}\|a\|_{L^{1}}\left(\int_{0}^{\frac{\left|x-x_{0}\right|}{2}} t d t\right)^{\frac{1}{2}} \sim\|a\|_{L^{1}} \frac{r^{M}}{\left|x-x_{0}\right|^{n+M}} .
\end{aligned}
$$


For $\mathrm{III}_{3}$, we have

$$
\begin{aligned}
I I I_{3} & =\left(\int_{\frac{\left|x-x_{0}\right|}{2}}^{\infty} \int_{B(x, t)}\left|\int_{B} Q_{t}(y, z) a(z) d z\right|^{2} \frac{d y d t}{t^{n+1}}\right)^{\frac{1}{2}} \\
& \lesssim_{M}\left(\int_{\frac{\left|x-x_{0}\right|}{2}}^{\infty} \int_{B(x, t)}\left(\int_{B} t^{-n}\left(\frac{\rho\left(x_{0}\right)}{t}\right)^{M}|a(z)| d z\right)^{2} \frac{d y d t}{t^{n+1}}\right)^{\frac{1}{2}} \\
& \lesssim_{M} \rho\left(x_{0}\right)^{M}\|a\|_{L^{1}}\left(\int_{\frac{\left|x-x_{0}\right|}{2}}^{\infty} \frac{d t}{t^{2 M+2 n+1}}\right)^{\frac{1}{2}} \sim\|a\|_{L^{1}} \frac{r^{M}}{\left|x-x_{0}\right|^{n+M}} .
\end{aligned}
$$

Thus, Case 3 has been solved.

To estimate $\|a\|_{L^{1}}$ in Proposition 3.1, we establish the following result without difficulty.

Lemma 3.1. Let $p>0, q \geq 1, \theta \geq 0$ and $\omega \in A_{q}^{\rho, \theta}$. For any $(p, q)_{\omega}$ weights $\omega(x)$ associated with $B=B\left(x_{0}, r\right)$,

$$
\|a\|_{L^{1}} \lesssim|B| \omega(B)^{-1 / p} \Psi(B)^{\theta},
$$

where the constant is exactly the $A_{q}^{\rho, \theta}$ weight constant of $w$.

Proof. If $q>1$, by Hölder inequality and the definition formula (2.3),

$$
\begin{aligned}
\|a\|_{L^{1}} & =\int_{B}|a(x)| w(x)^{1 / q} w(x)^{-1 / q} d x \\
& \leq\|a\|_{L^{q}(w)}\left(\int_{B} w(x)^{-q^{\prime} / q} d x\right)^{1 / q^{\prime}} \\
& \leq w(B)^{1 / q-1 / p}\left(\int_{B} w(x)^{-q^{\prime} / q} d x\right)^{1 / q^{\prime}}\left(\int_{B} w(x) d x\right)^{1 / q} w(B)^{-1 / q} \\
& \leq C|B| w(B)^{-1 / p} \Psi(B)^{\theta},
\end{aligned}
$$

where $C$ is the $A_{q}^{\rho, \theta}$ constant of $w$.

If $q=1$, we know that

$$
w(B) \leq C|B| \Psi(B)^{\theta} \operatorname{essinf}_{x \in B}\{w(x)\},
$$

by the definition formula (2.3), which means

$$
\left\|w^{-1}\right\|_{L^{\infty}(B)} \leq C|B| \Psi(B)^{\theta} w(B)^{-1},
$$

and thus

$$
\|a\|_{L^{1}} \leq\|a\|_{L^{1}(w)}\left\|w^{-1}\right\|_{L^{\infty}(B)} \leq C|B| w(B)^{-1 / p} \Psi(B)^{\theta} .
$$

All of the constants " $C$ " above are exactly the $A_{1}^{\rho, \theta}$ constant of $w$. 
Combining Proposition 3.1, Lemma 3.1 and noticing $\Psi(B)^{\theta} \lesssim_{\theta} 1$, the following result is obvious.

Corollary 3.1. Let $1 \leq q<\infty, \theta \geq 0, w \in A_{q}^{\rho, \theta}$. For any $(p, q)_{\omega}$ atoms a associated with $B=B\left(x_{0}, r\right)$ and all $x \in(4 B)^{c}$,

Case 1. if $\gamma_{0} r<\rho\left(x_{0}\right)$ and $\left|x-x_{0}\right| \leq 2 \rho\left(x_{0}\right)$,

$$
S_{Q} a(x) \lesssim w(B)^{-\frac{1}{p}}\left(\frac{r}{\left|x-x_{0}\right|}\right)^{n+\delta_{0}},
$$

Case 2. if $\gamma_{0} r<\rho\left(x_{0}\right)$ and $\left|x-x_{0}\right| \geq \rho\left(x_{0}\right)$, then for each $M>0$,

$$
S_{Q} a(x) \lesssim_{M} w(B)^{-\frac{1}{p}}\left(\frac{r}{\left|x-x_{0}\right|}\right)^{n+\delta_{0}}\left(\frac{\rho\left(x_{0}\right)}{\left|x-x_{0}\right|}\right)^{M},
$$

Case 3. if $\gamma_{0} r \geq \rho\left(x_{0}\right)$, then for each $M>0$,

$$
S_{Q} a(x) \lesssim M w(B)^{-\frac{1}{p}}\left(\frac{r}{\left|x-x_{0}\right|}\right)^{n+M} .
$$

The constant $\delta_{0}$ corresponds as in Lemma 2.2(ii).

Applying (iii) in Proposition 2.1 and Proposition 2.2, we can establish an analogue of Lemma 2.4 for $A_{q}^{\rho, \theta}$ weights, and thus the weighed version of Corollary 2.2 holds apparently. They will be used in the proof of Theorem 1.2 in Section 4.2.

Lemma 3.2. Let $1 \leq s, q<\infty, w \in A_{q}^{\rho, \theta}$ and $b \in B M O_{\theta}(\rho)$, then

$$
\left(\frac{1}{w(B)} \int_{B}\left|b-b_{B}\right|^{s} d w\right)^{1 / s} \lesssim\|b\|_{B M O_{\theta}(\rho)} \Psi(B)^{\eta_{0} / s+\theta^{\prime}}
$$

for all balls $B$, with $\eta_{0}=\eta_{0}(q)$ in Proposition 2.1(i) and $\theta^{\prime}$ in Lemma 2.4.

Proof. By homogeneity, we may assume $\|b\|_{B M O_{\theta}(\rho)}=1$ without loss of generality. For all balls $B$,

$$
\begin{aligned}
\frac{1}{w(B)} \int_{B}\left|b-b_{B}\right|^{s} d w & =s \int_{0}^{\infty} \lambda^{s-1} \frac{w\left(\left\{x \in B:\left|b(x)-b_{B}\right|>\lambda\right\}\right)}{w(B)} d \lambda \\
& \lesssim s \Psi(B)^{\eta_{0}} \int_{0}^{\infty}\left(\frac{\left|\left\{x \in B:\left|b(x)-b_{B}\right|>\lambda\right\}\right|}{|B|}\right)^{\delta} \lambda^{s-1} d \lambda \\
& \lesssim s \Psi(B)^{\eta_{0}} \int_{0}^{\infty} \exp \left(-\frac{c \lambda \delta}{\Psi(B)^{\theta^{\prime}}}\right) \lambda^{s-1} d \lambda \\
& =\Gamma(s+1) \Psi(B)^{\eta_{0}}\left(\frac{\Psi(B)^{\theta^{\prime}}}{c \delta}\right)^{s} \sim \Psi(B)^{\eta_{0}+s \theta^{\prime}}
\end{aligned}
$$

which yields the required inequality immediately. 
Corollary 3.2. Let $1 \leq s, q<\infty, w \in A_{q}^{\rho, \theta}$ and $b \in B M O_{\theta}(\rho)$, then

$$
\left(\frac{1}{w\left(2^{k} B\right)} \int_{2^{k} B}\left|b-b_{B}\right|^{s} d w\right)^{1 / s} \lesssim k\|b\|_{B M O_{\theta}(\rho)} \Psi\left(2^{k} B\right)^{\eta_{0} / s+\theta^{\prime}}
$$

for all balls $B$ and all $k \in \mathbb{N}^{*}$, with $\eta_{0}$ appears in Proposition 2.1(i) and $\theta^{\prime}$ appears in Lemma 2.4.

Proof. By using Lemma 3.2, the proof is quite similar to the proof of Lemma 1 in [1]. For each ball $B$,

$$
\left|b_{2 B}-b_{B}\right|=\left|f_{B}\left(b-b_{2 B}\right)\right| \leq \frac{|2 B|}{|B|} f_{2 B}\left|b-b_{2 B}\right| \leq 2^{n} \Psi(2 B)^{\theta}\|b\|_{B M O_{\theta}(\rho)} .
$$

Then we have

$$
\begin{aligned}
& \left(\frac{1}{w\left(2^{k} B\right)} \int_{2^{k} B}\left|b-b_{B}\right|^{s} d w\right)^{1 / s} \\
\leq & \left(\frac{1}{w\left(2^{k} B\right)} \int_{2^{k} B}\left|b-b_{2^{k} B}\right|^{s} d w\right)^{1 / s}+\sum_{j=1}^{k}\left|b_{2^{j} B}-b_{2^{j-1} B}\right| \\
\lesssim & \|b\|_{B M O_{\theta}(\rho)} \Psi\left(2^{k} B\right)^{\eta_{0} / s+\theta^{\prime}}+\sum_{j=1}^{k} \Psi\left(2^{j} B\right)^{\theta}\|b\|_{B M O_{\theta}(\rho)} \\
\lesssim & (k+1)\|b\|_{B M O_{\theta}(\rho)} \Psi\left(2^{k} B\right)^{\eta_{0} / s+\theta^{\prime}} .
\end{aligned}
$$

Since $1 \leq \Psi\left(2^{j} B\right) \leq \Psi\left(2^{k} B\right)$ and $\theta<\theta^{\prime}$. The proof is completed.

The authors [16] proved the following basic results.

Theorem 3.1. Let $1 \leq q<\infty, \theta \geq 0, w \in A_{q}^{\rho, \theta}$.

(i) If $q>1$, for all $f \in L_{w}^{q}\left(\mathbb{R}^{n}\right)$,

$$
\left\|S_{Q} f\right\|_{L_{w}^{q}} \lesssim\|f\|_{L_{w}^{q}}
$$

(ii) If $q=1$, for all $f \in L_{w}^{1}\left(\mathbb{R}^{n}\right)$,

$$
\left\|S_{Q} f\right\|_{W L_{w}^{1}} \lesssim\|f\|_{L_{w}^{1}}
$$

Theorem 3.2. Let $1<q<\infty, \theta \geq 0, w \in A_{q}^{\rho, \theta}$, then for all functions $f$ and $b \in B_{M O}(\rho)$,

$$
\left\|S_{Q, b} f\right\|_{L_{w}^{q}} \lesssim\|b\|_{B M O_{\theta}(\rho)}\|f\|_{L_{w}^{q}} .
$$




\section{Proof of main results}

\subsection{Proof of Theorem 1.1}

We prove Theorem 1.1(i) at first.

Proof of Theorem 1.1(i). Without loss of generality, we can assume $1<q<n\left(1+\delta_{0} / n\right)$. By Theorems 2.1 and 2.2, it needs only to prove that for all $(p, q)_{\omega}$ atoms or single $(p, q)_{\omega}$ atoms $a(x)$,

$$
\left\|S_{Q} a\right\|_{L^{p}(w)}^{p} \lesssim 1
$$

Firstly, if $a(x)$ is a single atom, the inequality above holds obviously. In fact, by Hölder inequality, Theorem 3.1(i) and the size condition of single atoms, we have

$$
\left\|S_{Q} a\right\|_{L_{w}^{p}} \leq\left\|S_{Q} a\right\|_{L_{w}^{q}} w\left(\mathbb{R}^{n}\right)^{1 / p-1 / q} \lesssim\|a\|_{L_{w}^{q}} w\left(\mathbb{R}^{n}\right)^{1 / p-1 / q} \leq 1
$$

Hence, it is sufficient for us to show that for any $(p, q)_{\omega}$ atoms $a(x)$ associated with a ball $B=B\left(x_{0}, r\right)$ and satisfies,

$$
\left\|S_{Q} a\right\|_{L^{p}(w)}^{p} \lesssim 1
$$

with the constant independent of $a(x)$.

We divide $\left\|S_{Q} a\right\|_{L_{w}^{p}}^{p}$ into two parts: $\left\|S_{Q} a\right\|_{L_{w}^{p}(4 B)}^{p}$ and $\left\|S_{Q} a\right\|_{L_{w}^{p}\left((4 B)^{c}\right.}^{p}$, where $B=B\left(x_{0}, r\right)$ is the ball $a(x)$ associated with.

For the first part, by Hölder inequality, $L_{w}^{q}$ boundedness of $S_{Q}$, the properties of $(p, q)_{\omega}$ atoms and Lemma 2.3,

$$
\begin{aligned}
\left\|S_{Q} a\right\|_{L_{w}^{p}(4 B)}^{p} & =\int_{4 B} S_{Q} a(x)^{p} w(x)^{\frac{p}{q}} w(x)^{\frac{q-p}{q}} d x \\
& \leq\left\|S_{Q} a\right\|_{L_{w}^{q}(4 B)}^{p} w(4 B)^{1-p / q} \\
& \lesssim\|a\|_{L_{w}^{q}}^{p} w(4 B)^{1-p / q} \leq\left(\frac{w(4 B)}{w(B)}\right)^{1-p / q} \\
& \lesssim\left(\frac{|4 B|}{|B|}\right)^{q-p} \Psi(4 B)^{\theta(q-p)} \lesssim 1 .
\end{aligned}
$$

The last inequality holds because $r \lesssim \rho\left(x_{0}\right)$.

For the second part, we denote the ring

$$
R B_{j}:=\left(2^{j} B\right) \backslash\left(2^{j-1} B\right) \quad \text { for } j \in \mathbb{N}^{*} .
$$

Consider the following two cases respectively: 
If $\gamma_{0} r \geq \rho\left(x_{0}\right)$ which means $r \sim \rho\left(x_{0}\right)$, by Case 3 in Lemma 3.1 and Lemma 2.3(iii), we have

$$
\begin{aligned}
\left\|S_{Q} a\right\|_{L_{w}^{p}\left((4 B)^{c}\right)}^{p} & =\int_{\left|x-x_{0}\right| \geq 4 r} S_{Q} a(x)^{p} w(x) d x \\
& \lesssim M \frac{1}{w(B)} \sum_{j=3}^{\infty} \int_{R B_{j}}\left(\frac{r}{\left|x-x_{0}\right|}\right)^{p(n+M)} w(x) d x \\
& \lesssim \frac{1}{w(B)} \sum_{j=3}^{\infty} 2^{-j p(n+M)} w\left(2^{j} B\right) \\
& \lesssim \sum_{j=3}^{\infty} 2^{-j p(n+M)}\left(2^{j n}\right)^{q}\left(1+\frac{2^{j} r}{\rho\left(x_{0}\right)}\right)^{q \theta} \\
& \lesssim \sum_{j=3}^{\infty} 2^{-j(p(n+M)-q n-q \theta)} .
\end{aligned}
$$

As long as we choose the positive constant $\mathrm{M}$ big enough such that $M>q(n+\theta) / p-n$, we obtain $\left\|S_{Q} a\right\|_{L_{w}^{p}\left((4 B)^{c}\right)}^{p} \lesssim 1$ in the case of $\gamma_{0} r \geq \rho\left(x_{0}\right)$.

If $\gamma_{0} r<\rho\left(x_{0}\right)$, for such a ball $B$, there exists $N_{0} \in \mathbb{N}$ satisfying $2^{N_{0}-1} r \leq \rho\left(x_{0}\right)<2^{N_{0}} r$. The second part

$$
\begin{aligned}
\left\|S_{Q} a\right\|_{L_{w}^{p}(4 B)^{c}}^{p} & =\left(\sum_{j=3}^{N_{0}}+\sum_{j=N_{0}+1}^{\infty}\right) \int_{R B_{j}} S_{Q} a(x)^{p} w(x) d x \\
& =: I_{1}+I_{2} .
\end{aligned}
$$

For $I_{1},\left|x-x_{0}\right|<2^{j} r \leq 2^{N_{0}} r \leq 2 \rho\left(x_{0}\right)$ implies $\Psi\left(2^{j} B\right) \leq 3$. By case in Lemma 3.1 and in Lemma 2.3(iii),

$$
\begin{aligned}
I_{1} & =\sum_{j=3}^{N_{0}} \int_{R B_{j}} S_{Q} a(x)^{p} w(x) d x \\
& \lesssim \frac{1}{w(B)} \sum_{j=3}^{N_{0}} \int_{R B_{j}}\left(\frac{r}{\left|x-x_{0}\right|}\right)^{p\left(n+\delta_{0}\right)} w(x) d x \\
& \lesssim \frac{1}{w(B)} \sum_{j=3}^{N_{0}} 2^{-j p\left(n+\delta_{0}\right)} w\left(2^{j} B\right) \\
& \lesssim \sum_{j=3}^{N_{0}} 2^{-j\left(p\left(n+\delta_{0}\right)-n q\right)} \lesssim 1 .
\end{aligned}
$$

The last inequality holds because of the assumption $q<p\left(1+\delta_{0} / n\right)$. 
For $I_{2},\left|x-x_{0}\right| \geq 2^{j-1} r \geq 2^{N_{0}} r>\rho\left(x_{0}\right)$ implies $\Psi\left(2^{j} B\right) \leq 2^{j+1} r / \rho\left(x_{0}\right)$. By case 2 in Lemma 3.1 and in Lemma 2.3(iii), for each $M>0$,

$$
\begin{aligned}
I_{2} & =\sum_{j=N_{0}+1}^{\infty} \int_{R B_{j}} S_{Q} a(x)^{p} w(x) d x \\
& \lesssim M \frac{1}{w(B)} \sum_{j=N_{0}+1}^{\infty} \int_{R B_{j}}\left(\frac{r}{\left|x-x_{0}\right|}\right)^{p\left(n+\delta_{0}\right)}\left(\frac{\rho\left(x_{0}\right)}{\left|x-x_{0}\right|}\right)^{M} w(x) d x \\
& \lesssim \sum_{j=N_{0}+1}^{\infty} 2^{-j p\left(n+\delta_{0}\right)} \frac{w\left(2^{j} B\right)}{w(B)}\left(\frac{\rho\left(x_{0}\right)}{2^{j} r}\right)^{M} \\
& \lesssim \sum_{j=N_{0}+1}^{\infty} 2^{-j\left(p\left(n+\delta_{0}\right)-n q\right)}\left(\frac{\rho\left(x_{0}\right)}{2^{j} r}\right)^{M-q \theta} \\
& \lesssim 1 \quad(\text { Let } M=q \theta) .
\end{aligned}
$$

Thus, the second part has the required estimate, too.

Now we turn to (ii) in Theorem 1.1. Firstly, we need the following Lemma.

Lemma 4.1. Let $w \in A_{1}^{\rho, \infty}$ and $0<p<1$. If a sequence measurable functions $\left\{f_{j}\right\}$ satisfy

$$
\left\|f_{j}\right\|_{W L_{w}^{p}} \leq 1, \quad \forall j \in \mathbb{Z} \quad \text { and } \quad \sum_{j \in \mathbb{Z}}\left|\lambda_{j}\right|^{p} \leq 1
$$

then we have

$$
\left\|\sum_{j \in \mathbb{Z}} \lambda_{j} f_{j}\right\|_{W L_{w}^{p}}^{p} \leq \frac{2-p}{1-p} .
$$

The proof can be found in [9].

Proof of Theorem 1.1(ii). By Lemma 4.1, we need only to prove the following inequality

$$
\sup _{\lambda>0} \lambda^{p} w\left(\left\{x \in \mathbb{R}^{n}:\left|S_{Q}(a)(x)\right|>\lambda\right\}\right) \lesssim 1
$$

holds for any atom $a(x)$.

If $a(x)$ is a single atom, then

$$
\sup _{\lambda>0} \lambda^{p} w\left(\left\{x \in \mathbb{R}^{n}:\left|S_{Q}(a)(x)\right|>\lambda\right\}\right) \leq\left\|S_{Q} a\right\|_{L_{w}^{p}(4 B)}^{p} \lesssim 1 .
$$

Therefore, it is sufficient for us to prove that for any $\lambda>0$ and any a $(p, q)_{\omega}$ atom $a(x)$, associated with a ball $B=B\left(x_{0}, r\right), \lambda^{p} w\left(E_{\lambda}\right) \lesssim 1$, where the constant does not dependent on $\lambda$ and $a(x)$, and

$$
E_{\lambda}=\left\{x \in \mathbb{R}^{n}:\left|S_{Q}(f)(x)\right|>\lambda\right\} .
$$


Divide $E_{\lambda}$ into

$$
E_{\lambda}=E_{\lambda}^{\prime} \bigcup\left(\bigcup_{j=3}^{\infty} E_{\lambda, j}\right),
$$

where

$$
E_{\lambda}^{\prime}=E_{\lambda} \bigcap 4 B, \quad E_{\lambda, j}=E_{\lambda} \bigcap R B_{j} .
$$

As the argument in the proof of Theorem 1.1(i), we still have $\left\|S_{Q} a\right\|_{L_{w}^{p}(4 B)}^{p} \lesssim 1$. Therefore, by Chebshev inequality,

$$
\lambda^{p} w\left(E_{\lambda}^{\prime}\right) \leq\left\|S_{Q} a\right\|_{L_{w}^{p}(4 B)}^{p} \lesssim 1
$$

For $E_{j, \lambda}$, we are going to prove there exists a constant $c_{0}>0$ independent of $j$ and the selection of " $a(x)$ ", such that

$$
S_{Q} a(x) \leq c_{0} w\left(2^{j} B\right)^{-1 / p} .
$$

Similarly as the proof of (i) in Theorem 1.1, To achieve the goal, we need to consider the condition $\gamma_{0} r<\rho\left(x_{0}\right)$ and $\gamma_{0} r \geq \rho\left(x_{0}\right)$ respectively. But we will consider the condition $\gamma_{0} r<\rho\left(x_{0}\right)$ only. In fact, in Corollary 3.1, the conclusion in case 3 is stronger, so the condition $\gamma_{0} r \geq \rho\left(x_{0}\right)$ is easier then that of $\gamma_{0} r<\rho\left(x_{0}\right)$, and we can follow the proof of the latter without difficulty. The proof of (i) in Theorem 1.1 can also corroborate the former assertion.

When $\gamma_{0} r<\rho\left(x_{0}\right)$, we consider the case $j \leq N_{0}$ and $j \geq N_{0}+1$ respectively, where the integer $N_{0}$ is defined by $2^{N_{0}-1} \leq \rho\left(x_{0}\right)<2^{N_{0}}$ as in the proof of (i) in Theorem 1.1.

If $j \leq N_{0}$, which means $\left|x-x_{0}\right|<2^{j} r \leq 2^{N_{0}} r \leq 2 \rho\left(x_{0}\right)$ and $\Psi\left(2^{j} B\right) \leq 3$, by case 2 in Lemma 3.1 and Lemma 2.3(iii), we have

$$
\begin{aligned}
S_{Q} a(x) & \lesssim w(B)^{-\frac{1}{p}}\left(\frac{r}{\left|x-x_{0}\right|}\right)^{n+\delta_{0}} \\
& \lesssim w\left(2^{j} B\right)^{-1 / p} 2^{-j\left(n+\delta_{0}\right)}\left(\frac{w\left(2^{j} B\right)}{w(B)}\right)^{1 / p} \\
& \lesssim w\left(2^{j} B\right)^{-1 / p} 2^{-j\left(n+\delta_{0}\right)} 2^{j / p} \Psi\left(2^{j} B\right)^{\theta / p} \\
& \lesssim w\left(2^{j} B\right)^{-1 / p} 2^{j\left(-n-\delta_{0}+n / p\right)} \\
& =w\left(2^{j} B\right)^{-1 / p},
\end{aligned}
$$

where all constants do not depend on $j$ and $a(x)$.

If $j \geq N_{0}+1$, which means

$$
\left|x-x_{0}\right| \geq 2^{j-1} r \geq 2^{N_{0}} r>\rho\left(x_{0}\right) \quad \text { and } \quad \Psi\left(2^{j} B\right) \leq 2^{j+1} r / \rho\left(x_{0}\right),
$$


by Case 2 in Lemma 3.1 and Lemma 2.3(iii), we have

$$
\begin{aligned}
S_{Q} a(x) & \lesssim w(B)^{-\frac{1}{p}}\left(\frac{r}{\left|x-x_{0}\right|}\right)^{n+\delta_{0}}\left(\frac{\rho\left(x_{0}\right)}{\left|x-x_{0}\right|}\right)^{M} \\
& \lesssim w\left(2^{j} B\right)^{-1 / p} 2^{-j\left(n+\delta_{0}\right)}\left(\frac{\rho\left(x_{0}\right)}{2^{j} r}\right)^{M}\left(\frac{w\left(2^{j} B\right)}{w(B)}\right)^{1 / p} \\
& \lesssim w\left(2^{j} B\right)^{-1 / p} 2^{-j\left(n+\delta_{0}\right)}\left(\frac{\rho\left(x_{0}\right)}{2^{j} r}\right)^{M} 2^{j n / p} \Psi\left(2^{j} B\right)^{\theta / p} \\
& \lesssim w\left(2^{j} B\right)^{-1 / p}\left(\frac{\rho\left(x_{0}\right)}{2^{j} r}\right)^{M-\theta / p} \\
& =w\left(2^{j} B\right)^{-1 / p} \quad(\text { Let } M=\theta / p),
\end{aligned}
$$

where all constants do not depend on $j$ and $a(x)$, either. Therefore, formula (4.2) holds.

To end the proof, we now consider two cases about $j$.

Case 1 . There exists a $j \in \mathbb{N}$ such that

$$
c_{0} w\left(2^{j} B\right)^{-1 / p} \leq \lambda
$$

So there exists a maximum integer $K=K(\lambda)$ (if no exists such $K$, there is nothing to prove) such that

$$
c_{0} w\left(2^{K} B\right)^{-1 / p}>\lambda,
$$

which means for each $j \geq K+1, C_{0} w\left(2^{j} B\right)^{-1 / p} \leq \lambda$ and therefore $E_{\lambda, j}=\varnothing$. We may assume $K>0$, since otherwise, $E_{\lambda, j}=\varnothing$ for all $j \in \mathbb{N}^{*}$ and $E_{\lambda}=E_{\lambda}^{\prime}$. When $K>0$, we have

$$
\lambda^{p} w\left(E_{\lambda} \backslash E_{\lambda}^{\prime}\right)=\lambda^{p} \sum_{j=3}^{\infty} w\left(E_{\lambda, j}\right) \leq C_{0}^{p} w\left(2^{K} B\right)^{-1} \sum_{j=1}^{K} w\left(E_{\lambda, j}\right) \leq C_{0}^{p}
$$

Case 2. For any $j \in \mathbb{N}$ such that

$$
c_{0} w\left(2^{j} B\right)^{-1 / p} \geq \lambda .
$$

So

$$
c_{0} w\left(\mathbb{R}^{n}\right)^{-1 / p} \geq \lambda
$$

Thus,

$$
\lambda^{p} w\left(E_{\lambda}\right) \leq c_{0}^{p} w\left(\mathbb{R}^{n}\right)^{-1} w\left(\mathbb{R}^{n}\right) \lesssim 1 .
$$

Combining (4.1), (4.3) and (4.4), the required inequality holds. 


\subsection{Proof of Theorem 1.2}

We are going to prove Theorem 1.2 in this section.

Proof of Theorem 1.2. We may assume $\|b\|_{B \mathrm{BO}_{\theta}(\rho)}=1$ by homogeneity. Suppose $f=$ $\sum_{j \in \mathbb{N}} \lambda_{j} a_{j}$, where $\sum_{j \in \mathbb{N}}\left|\lambda_{j}\right|=1, a_{0}$ is a $(1, q)_{\omega}$ single atom, and $a_{j}(j \geq 1)$ is a $(1, q)_{\omega}$ atom supported on $B_{j}$, where $1<q<\infty$. Let $b_{j}=b_{B_{j}} \in \mathbb{R}$, it is sufficient for us to prove that for all functions $f$ satisfying the prerequisites above,

$$
\left\|S_{Q, b} f\right\|_{W L_{w}^{1}} \lesssim 1
$$

By the sublinearity of $S_{Q}$,

$$
\begin{aligned}
S_{Q, b} f(x) & =S_{Q}((b(x)-b) f)(x)=S_{Q}\left((b(x)-b) \sum_{j \in \mathbb{N}} \lambda_{j} a_{j}\right)(x) \\
& =S_{Q}\left(\lambda_{0}(b(x)-b) a_{0}+\sum_{j \in \mathbb{N}^{*}} \lambda_{j}\left(b_{j}-b\right) a_{j}+\sum_{j \in \mathbb{N}^{*}} \lambda_{j}\left(b(x)-b_{j}\right) a_{j}\right)(x) \\
& \leq\left|\lambda_{0}\right| S_{Q, b} a_{0}(x)+S_{Q}\left(\sum_{j \in \mathbb{N}^{*}} \lambda_{j}\left(b_{j}-b\right) a_{j}\right)(x)+\sum_{j \in \mathbb{N}^{*}}\left|\lambda_{j}\right|\left|b(x)-b_{j}\right| S_{Q} a_{j}(x) \\
& :=\left|\lambda_{0}\right| S_{Q, b} a_{0}(x)+S_{Q}\left(\sum_{j \in \mathbb{N}^{*}} \lambda_{j} A_{j}\right)(x)+\sum_{j \in \mathbb{N}^{*}}\left|\lambda_{j}\right| B_{j}(x),
\end{aligned}
$$

where

$$
A_{j}(x)=\left(b_{j}-b(x)\right) a_{j}(x), \quad B_{j}(x)=\left|b(x)-b_{j}\right| S_{Q} a_{j}(x) \geq 0 .
$$

By the formula above and Theorem 3.1(ii), and noticing $\sum_{j \in \mathbb{N}}\left|\lambda_{j}\right|=1$,

$$
\begin{aligned}
\left\|S_{Q, b} f\right\|_{W L_{w}^{1}} & \lesssim\left|\lambda_{0}\right|\left\|S_{Q, b} a_{0}(x)\right\|_{W L_{w}^{1}}+\left\|S_{Q}\left(\sum_{j \in \mathbb{N}^{*}} \lambda_{j} A_{j}\right)\right\|_{W L_{w}^{1}}+\left\|\sum_{j \in \mathbb{N}^{*}}\left|\lambda_{j}\right| B_{j}(x)\right\|_{W L_{w}^{1}} \\
& \lesssim\left|\lambda_{0}\right|\left\|S_{Q, b} a_{0}(x)\right\|_{L_{w}^{1}}+\left\|\sum_{j \in \mathbb{N}^{*}} \lambda_{j} A_{j}\right\|_{L_{w}^{1}}+\left\|\sum_{j \in \mathbb{N}^{*}}\left|\lambda_{j}\right| B_{j}(x)\right\|_{L_{w}^{1}} \\
& \leq \sup _{j}\left\{\left\|S_{Q, b} a_{0}(x)\right\|_{L_{w}^{1}},\left\|A_{j}\right\|_{L_{w}^{1}},\left\|B_{j}\right\|_{L_{w}^{1}}\right\} .
\end{aligned}
$$

Hence, in order to prove formula (4.5), we just need to prove that for all $b \in B M O_{\theta}(\rho)$ satisfying $\|b\|_{B M O_{\theta}(\rho)}=1$, single atoms $a_{0}$, and $(1, q)_{\omega}$ atoms $a$ supported on $B=B\left(x_{0}, r\right)$, the following three formulae holds:

$$
\begin{aligned}
& \left\|S_{Q, b} a_{0}\right\|_{L_{w}^{1}} \lesssim 1 \\
& \left\|\left(b-b_{B}\right) a\right\|_{L_{w}^{1}} \lesssim 1 \\
& \left\|\left(b-b_{B}\right) S_{Q} a\right\|_{L_{w}^{1}} \lesssim 1 .
\end{aligned}
$$


Firstly, we consider the case of single atoms, namely formula (4.6a). By Hölder inequality, Theorem 1.1 and the size condition of single atoms,

$$
\left\|S_{Q, b} a_{0}\right\|_{L_{w}^{1}} \leq\left\|S_{Q, b} a_{0}\right\|_{L_{w}^{q}}\|1\|_{L_{w}^{q^{\prime}}} \lesssim\left\|a_{0}\right\|_{L_{w}^{q}} w\left(\mathbb{R}^{n}\right)^{1 / q^{\prime}} \leq w\left(\mathbb{R}^{n}\right)^{1 / q-1} w\left(\mathbb{R}^{n}\right)^{1 / q^{\prime}}=1,
$$

which means formula (4.6a) holds.

For formula (4.6b), By (ii) in Theorem 3.2(ii), Hölder inequality, Lemma 3.2, and noticing $\Psi(B) \leq 3$,

$$
\begin{aligned}
& \left\|S_{Q}\left(\left(b-b_{B}\right) a\right)\right\|_{W L_{w}^{1}} \leq\left\|\left(b-b_{B}\right) a\right\|_{L_{w}^{1}} \\
& \leq\left\|b-b_{B}\right\|_{L_{w}^{q^{\prime}(B)}}\|a\|_{L_{w}^{q}} \\
& \lesssim w(B)^{1 / q^{\prime}} \Psi(B)^{\eta_{0} / s+\theta_{0}} w(B)^{1 / q-1} \\
& \lesssim 1 \text {. }
\end{aligned}
$$

Therefore, formula (4.6b) holds.

Finally, we will complete the proof of formula (4.6c), whose basic idea is similarly as Theorem 1.1(i). Similarly as the discussion in the proof of (4.2), we will just consider the condition $\gamma_{0} r<\rho\left(x_{0}\right)$.

Suppose $\gamma_{0} r<\rho\left(x_{0}\right)$, we also note $N_{0} \in \mathbb{Z}$ satisfying $2^{N_{0}-1} r \leq \rho\left(x_{0}\right)<2^{N_{0}} r$, and We may assume $N_{0} \geq 3$. Let $I$ is the left side of formula (4.6c), and decompose $I$ as follow:

$$
\begin{aligned}
I= & \int_{4 B}\left|b(x)-b_{B}\right| S_{Q} a(x) d w+\sum_{j=3}^{N_{0}} \int_{R B_{j}}\left|b(x)-b_{B}\right| S_{Q} a(x) d w \\
& \quad+\sum_{j=N_{0}}^{\infty} \int_{R B_{j}}\left|b(x)-b_{B}\right| S_{Q} a(x) d w \\
= & : I_{1}+I_{2}+I_{3} .
\end{aligned}
$$

For $I_{1}$, noticing $\Psi(4 B) \leq 9$, by Hölder inequality, (i) in Theorem 3.1, the size condition of $a$, (iii) in Lemma 2.3 and Corollary 3.2,

$$
\begin{aligned}
& I_{1} \leq\left\|b-b_{B}\right\|_{L_{w}^{q^{\prime}(4 B)}}\left\|S_{Q} a\right\|_{L_{w}^{q}} \lesssim\left\|b-b_{B}\right\|_{L_{w}^{q^{\prime}(B)}}\|a\|_{L_{w}^{q}} \\
& \lesssim w(4 B)^{1 / q^{\prime}} \Psi(4 B)^{\eta_{0} / s+\theta_{0}} w(B)^{1 / q-1} \\
& \lesssim\left(\frac{|4 B|}{|B|}\right)^{q / q^{\prime}} \Psi(4 B)^{\theta q / q^{\prime}+\eta_{0} / s+\theta_{0}} \\
& \lesssim 1 \text {. }
\end{aligned}
$$

For $I_{2}$ where $j \leq N_{0}$, we have $\left|x-x_{0}\right|<2^{j} r \leq 2^{N_{0}} r \leq 2 \rho\left(x_{0}\right)$ and $\Psi\left(2^{j} B\right) \leq 3$. By case 1 
of Lemma 3.1, (iii) in Lemma 2.3 and Corollary 3.2,

$$
\begin{aligned}
I_{2} & =\sum_{j=3}^{N_{0}} \int_{R B_{j}}\left|b(x)-b_{B}\right| S_{Q} a(x) d w \\
& \lesssim \sum_{j=3}^{N_{0}} 2^{-j\left(n+\delta_{0}\right)} w(B)^{-1} \int_{2^{j} B}\left|b-b_{B}\right| d w \\
& \lesssim \sum_{j=3}^{N_{0}} 2^{-j\left(n+\delta_{0}\right)} \frac{w\left(2^{j} B\right)}{w(B)} \frac{1}{w\left(2^{j} B\right)} \int_{2^{j} B}\left|b-b_{B}\right| d w \\
& \lesssim \sum_{j=3}^{N_{0}} j 2^{-j\left(n+\delta_{0}-n q\right)} \Psi\left(2^{j} B\right)^{q \theta+\eta_{0}+\theta_{0}} \\
& \lesssim \sum_{j=1}^{\infty} j 2^{-j\left(n+\delta_{0}-n q\right)} \\
& \lesssim 1 \quad\left(\text { since } q<1+\delta_{0} / n\right) .
\end{aligned}
$$

For $I_{3}$, we have $\left|x-x_{0}\right| \geq 2^{j-1} r \geq 2^{N_{0}} r>\rho\left(x_{0}\right)$, and $\Psi\left(2^{j} B\right) \leq 2^{j+1} r / \rho\left(x_{0}\right)$. Consequently,

$$
\begin{aligned}
I_{3} & =\sum_{j=N_{0}+1}^{\infty} \int_{R B_{j}}\left|b(x)-b_{B}\right| S_{Q} a(x) d w \\
& \lesssim M \sum_{j=N_{0}+1}^{\infty} 2^{-j\left(n+\delta_{0}\right)}\left(\frac{\rho\left(x_{0}\right)}{2^{j} r}\right)^{M} w(B)^{-1} \int_{2^{j} B}\left|b-b_{B}\right| d w \\
& \lesssim \sum_{j=N_{0}+1}^{\infty} 2^{-j\left(n+\delta_{0}\right)}\left(\frac{\rho\left(x_{0}\right)}{2^{j} r}\right)^{M} \frac{w\left(2^{j} B\right)}{w(B)} \frac{1}{w\left(2^{j} B\right)} \int_{2^{j} B}\left|b-b_{B}\right| d w \\
& \lesssim \sum_{j=N_{0}+1}^{\infty} j 2^{-j\left(n+\delta_{0}-n q\right)}\left(\frac{\rho\left(x_{0}\right)}{2^{j} r}\right)^{M} \Psi\left(2^{j} B\right)^{q \theta+\eta_{0}+\theta_{0}} \\
& \lesssim \sum_{j=N_{0}+1}^{\infty} j 2^{-j\left(n+\delta_{0}-n q\right)}\left(\frac{\rho\left(x_{0}\right)}{2^{j} r}\right)^{M-\left(q \theta+\eta_{0}+\theta_{0}\right)} \\
& \lesssim 1 \quad\left(M=q \theta+\eta_{0}+\theta_{0}\right) .
\end{aligned}
$$

Therefore, we have proved formula (4.6c) under the condition $\gamma_{0} r<\rho\left(x_{0}\right)$. For the condition $\gamma_{0} r \geq \rho\left(x_{0}\right)$, as what we have discussed, the proof is easier, so formula (4.6c) holds.

In summarize, formulae (4.6a)-(4.6c) hold, so formula (4.5) holds and we have proved the required conclusion. 


\section{Acknowledgements}

The research was supported by the NNSF (No. 11771023) of China.

\section{References}

[1] B. Bongioanni, E. Harboure and O. Salinas, Commutators of Riesz transforms related to Schrödinger operators, J. Fourier Anal Appl., 17 (2011), 115-134.

[2] B. Bongioanni, E. Harboure and O. Salinas, Class of weights related to Schrödinger operators, J. Math. Anal. Appl., 373 (2011), 563-579.

[3] J, Duoandikoetxea, Fourier Analysis, Grad. Stud. Math. Soc., Providence, 2000.

[4] J. Dziubański and J. Zienkiewicz, Hardy space $H^{1}$ associated to Schrödinger operator with potential satisfying reverse Hölder inequality, Rev. Math. Iber., 15 (1999), 279-296.

[5] J. Dziubański, G. Garrigós, J. Torrea and J. Zienkiewicz, BMO spaces related to Schrödinger operators with potentials satisfying a reverse Hölder inequality, Math. Z., 249 (2005), 249356.

[6] J. García-Cuerva and J. Rubio de Francia, Weighted Norm Inequalities and Related Topics, Amsterdam-New York, North-Holland, 1985.

[7] S. Hartzstein, and O. Salinas, Weighted $B M O$ and Carleson measures on spaces of homogeneous, J. Math. Anal. Appl., 342 (2008), 950-969.

[8] H. Liu, L. Tang and H. Zhu, Weighted Hardy spaces and BMO spaces associated with Schrödinger operators, Math. Nachr., 285 (2012), 2173-2207.

[9] S. Lu, Four Lectures on Real $H^{p}$ Spaces, Word Scientific Publishing, Riveredge, N. J., 1995.

[10] B. Muckenhoupt, Weighted norm inequalities for the Hardy maximal functions, Trans. Amer. Math. Soc., 165 (1972), 207-226.

[11] C. Pérez, Endpoint estimates for commutators of singular integral operators, J. Funct. Anal., 128 (1995), 163-185.

[12] Z. Shen, $L^{p}$ estimates for Schrödinger operators with certain potentials, Ann. Inst. Fourier. Grenoble., 45 (1995), 513-546.

[13] L. Song and L. X. Yan, Riesz transforms associated to Schrödinger operators on weighted Hardy spaces, J. Funct. Anal., 259 (2010), 1466-1490.

[14] L. Tang, Weighted norm inequalities for Schrödinger type operators, Forum Math., 27 (2015), 2491-2532.

[15] L. Tang, Weighted norm inequalities for commutators of Littlewood-Paley functions related to Schrödinger operators, arXiv:1109.0100.

[16] L. Tang, J. Wang and H. Zhu, Weighted norm inequalities for area functions related to Schrödinger operators, Izv. Nats. Akad. Nauk Armenii Mat., 54 (2019), 40-59.

[17] H. Zhu and L. Tang, Weighted local Hardy spaces associated to Schrödinger operators, Illinois J. Math., 60 (2016), 687-738.

[18] D. Yang and Y. Zhou, Localized Hardy spaces $H^{1}$ related to admissible functions on RDspaces and applications to Schrödinger operators, Trans. Amer. Math. Soc., 363 (2011), 11971239.

[19] J. Zhong, Harmonic Analysis for Some Schrödinger Type Operators, Ph.D. Thesis, Princeton University, 1993. 